\title{
Simulation of 3D Ultrasound with a Realistic Electro-mechanical Model of the Heart
}

\author{
Q. Duan ${ }^{1}$, P. Moireau ${ }^{2}$, E.D. Angelini ${ }^{3}$, D. Chapelle ${ }^{2}$, and A.F. Laine ${ }^{1}$ \\ ${ }^{1}$ Dpt. of Biomedical Eng., Columbia University, NY, USA \\ ${ }^{2}$ Project MACS, INRIA-Rocquencourt, France \\ ${ }^{3}$ Dpt. TSI, GET-Ecole Nationale Supérieure des Télécommunications, LTCI \\ CNRS-UMR 5141, France
}

\begin{abstract}
This paper presents a first set of experiments to integrate a realistic electro-mechanical model of a beating heart into simulated real-time three-dimensional (RT3D) ultrasound data. A novel ultrasound simulation framework is presented, extended from the model of Meunier [12. True three-dimensional transducer modeling was performed, using RT3D acquisition design. Myocardium and blood scattering parameters were defined in three dimensions. Ultrasound data sets were generated for a normal case and a pathological case, simulating left bundle branch block. Accuracy of an optical flow tracking method was evaluated on the simulated data to measure displacements on the myocardial surfaces and inside the myocardium over a cardiac cycle. The proposed simulation framework has important motivations in a cardiac modeling context as part of this project is focused on the design of effective parameter estimation methods, based on cardiac imaging.
\end{abstract}

\section{Introduction}

Ultrasound is the cardiac screening modality with the highest temporal resolution, but is still limited to two-dimensions in most hospitals and clinical centers. Development of 3D echocardiography started in the 1990s [23], with real-time 3D (RT3D) ultrasound based on matrix phased arrays. A new generation of RT3D transducers was introduced by Duke University and more recently by Philips Medical Systems (Best, The Netherlands) with the SONOS 7500 transducer followed by the IE33 model that acquires a fully sampled cardiac volume within four cardiac cycles. This technical advance increased the spatial resolution and image quality, which makes 3D ultrasound techniques increasingly attractive for quantitative cardiac diagnosis on patients 21]. Since RT3D ultrasound acquires volumetric ultrasound sequences with fairly high temporal resolution using a fixed-positioned transducer, it can capture complex 3D cardiac motion very well. Cardiac motion analysis from clinical image sequences has been an active research area over the past decade. Previous efforts using ultrasound data for motion analysis include intensity-based speckle tracking and optical flow (OF), or dedicated imaging set ups such as strain-imaging, and elastography. In [16] for example, a shape tracking approach was used to track endocardial surface 
patches in $3 \mathrm{D}$ echocardiography for motion analysis. In a previous study [87] we developed a framework to derive several dynamic cardiac measures based on four-dimensional optical flow using RT3D ultrasound sequences. This framework was applied to a clinical data set from a heart transplant patient and the dynamic measurements, computed with this method, agreed with findings in other cardiac biomechanics studies.

In this paper, in order to quantitatively evaluate our tracking method, we present a framework to generate 3D simulated ultrasound data using a realistic electro-mechanical model of a human heart, namely, a model that generates displacement and deformation patterns - as well as global physiological indicators such as pressures and flows - representative of clinical observations. A numerical cardiac phantom was used to mimic realistic anatomy, along with acoustic impedance of cardiac tissue and blood, as well as motion field of the myocardium during a cardiac cycle. Based on this phantom, simulated linear three-dimensional ultrasound data sets were generated, using similar transducer parameters as in the RT3D ultrasound machine from Volumetrics (Durham, NC). Details of the 3D ultrasound simulation framework as well as performance of our optical flow based method in tracking myocardial tissues were explored by simulating consecutive RT3D ultrasound frames of the beating heart under known motion fields, including translation, rotation, and thickening deformations. Our approach also has important motivations in a cardiac modeling context. Indeed, part of this project is focused on the design of effective parameter estimation methods - relevant for diagnosis assistance - based on clinical measures and simulations from a mathematical model of the beating heart [17/21]. Difficulties encountered in such estimation fall into two main categories:

- Modeling difficulties, namely, the mathematical model must accurately represent actual phenomena observed in clinical measurements and reflect pathologies of interest targeted in a diagnosis assistance setup; and

- Estimation difficulties, namely, the parameter estimation procedure per se must be carefully designed in order to be effective when the model is adequate, taking into account the presence of measurement errors.

Therefore, when focusing on the design of a parameter estimation strategy for a given class of models, one crucial step consists in generating synthetic measurements from the model for a specific choice of parameters. Indeed, by doing so we can circumvent modeling difficulties. The parameter estimation procedure is then assessed by processing these synthetic measurements, combined with well-chosen measurement noise components, and extracting modeling parameters to be compared with the initial values. Of course, for this assessment to be meaningful, the generation of synthetic measurements must be carefully designed, close to physiological conditions, while the processing of these measurements must take into account the constraints and uncertainties associated with a given modality of observation (e.g. cardiac imaging). In this context, this paper presents a first series of experimentation integrating our cardiac model into 3D echocardiographic data sets. 


\section{Electro-mechanical Model of the Heart}

\subsection{Heart Anatomy}

We use the "Generic Anatomical Heart Model" presented in [21, which approximates the ventricles by truncated ellipsoids This generic model could be adapted to specific patient anatomies by fitting the model geometry to medical image segmentation results - in particular when pursuing the above-mentioned estimation and diagnosis goal - but this was not needed for the present study.

\subsection{Bio-physical Properties of the Model}

The electro-mechanical model employed to generate realistic deformation patterns of the heart during a complete cycle was fully described in [17. The underlying contraction law is based on physiological considerations and satisfies essential thermo-mechanical requirements. This law incorporates contractility parameters that can be varied to simulate cardiac pathologies such as infarcts. Electrical activation is modeled based on the bidomain equations [15, with action potential propagation initiated near the apex on the endocardial surface. Blood inside each ventricle is modeled as a simplified lumped pressure / volume system, and cardiac cycle phases are distinguished through coupling conditions between the internal fluid and the different compartments of the cardiovascular system - in particular systemic and pulmonary circulations, both represented with Windkessel models. Numerical methods - including finite element procedures - used to obtain simulations of this global heart model were also described in [17. Simulation results are illustrated in Fig 1, where we compared simulation of a normal heart (our reference) with results corresponding to a left bundle branch block (LBBB) modeled via initiation of the electrical activation in the right ventricle only.
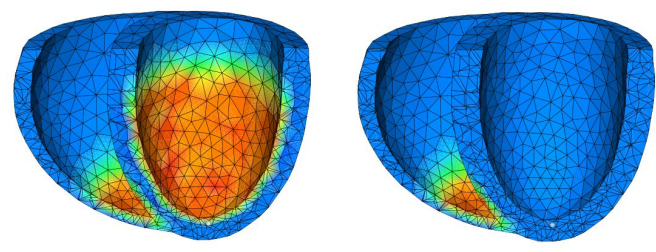

Fig. 1. Electrical activation at early stage: Reference (left) and LBBB (right)

\subsection{Motion Field Generation and Format}

We used several numerical simulation results with the model described above to generate rasterized 3D images of the cardiac tissue over a complete cycle, with a spatial sampling of $1 \mathrm{~mm}^{3}$ and a temporal sampling of $5 \mathrm{~ms}$, comparable to $3 \mathrm{D}$ echography sampling capabilities. We recall that the rasterization of an 


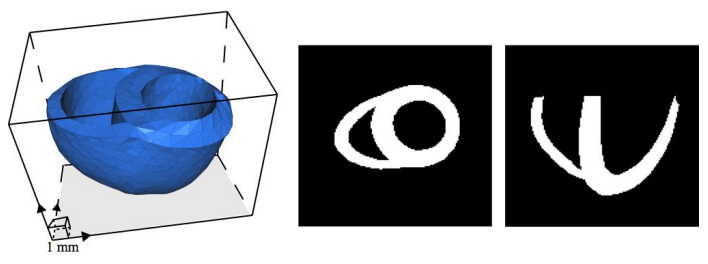

Fig. 2. Myocardial mesh and corresponding rasterization during systole

object consists in computing the set of voxels enclosed within the object boundaries. The algorithm used is described in 20, see also 4. In Fig. 2, we show cross-section views of these images during systole. The 3D ultrasound simulation procedure takes these images as an input, and optical flow tracking results can then be assessed using the reference values of the displacements between two subsequent images, whose exact values can be obtained for each voxel by simple post-processing of the model simulations.

The physics of ultrasound imaging being rather well-known, accurate simulations of the measurements can be obtained, including for 3D echography [9]. Hence, the remaining key point in the assessment of estimation procedures concerns the processing step leading to data used as an input in the estimation, such as the optical flow that provides displacements or velocities, for which the corresponding errors must be accurately determined.

\section{3D Ultrasound Simulation}

\subsection{Review of Existing Methods}

Simulating ultrasonic B-scanning images can be dated back to 1980 [3. In their paper, Bamber et al. modeled the ultrasonic image formation process in the far-field as a convolution of the point spread function from the transducer and the scattered distribution of underlining tissues defined by local density and compressibility.

Several studies have been performed since then, with more or less elaborate models. In [14, Narayanan et al. used a very simple simulation framework for transmitted pulses and received echo signals to study the influence of the scattering cross-section and the number of scatterers per cells on the envelope signal statistics. Their model was derived from the extensive review of first and second order statistics of complex random signals from Wagner et al. 27]. Proposing to model the scattering cross-section as a Gamma random variable, and setting the number of scatterers to 7, 13 and 38 they showed strong deviation from a Rayleigh distribution as randomness of the cross sections increased and number of scatterers decreased.

In the field of specific cardiac applications, a study of reference in [5] demonstrated that the K-distribution is generally more appropriate than the Rayleigh distribution to simulate backscatters from myocardial tissue in the frequency 
range of 5-15 MHz. The K-distribution is generated by a sparse density of Poisson scatterers (average number of scatterers per resolution cell $\rho$ between 9 and 12), whereas the Rayleigh distribution is generated by a dense distribution of Poisson scatterers ( $\rho$ above 12) [2515].

In a seminal paper [12, Meunier and Bertrand proposed a three-dimensional inhomogeneous continuum model to mimic myocardial tissue as a collection of acoustic cells with individual impedance. Their work was motivated by the need to understand mean gray level changes occurring with different tissue motions. An important assumption was made in their work, assuming small and dense scatterers per resolution cells, leading to Rayleigh distributed amplitudes of the envelope signals. In [13, the same authors looked at the problem of decorrelation between tissue and speckle patterns under linear transformations (2D rotation, translation and deformation). They identified threshold values of deformation amplitudes to guarantee visual correlation of backscattered patterns between two consecutive ultrasound frames.

Jensen [9] developed an extensive ultrasound simulation software called FIELD in 1996 based on Tupholme-Stepanishen's method, which simulates acoustic fields generated from arbitrarily designed transducers. Modeling is based on the numerical computation of a transmit excitation function (e.g. sine wave with Gaussian envelope) with the spatial impulse response of the transducer, which is the sum of small individual array elements, organized according to a pre-defined geometry.

A 3D extension of the model,generating 3D ultrasound image volume can also be simulated, as shown in [1] and 30] most recently.

Regarding the three-dimensional aspect of the ultrasound simulation problem, a first approach was proposed by [18 to simulate acoustic fields generated by phased array transducers, with waves propagating in a uniformly lossy media. Their approach was based on a double integral of the Huygens-Fresnel equations to describe the spatial behavior of the wave front pressure in the acoustic field, generated from a matrix of transducers. Later, with the introduction of the Volumetrics RT3D transducer from Smith and Von Ramm, several papers 22,29|28 were published, performing similar simulations for different geometrical designs of the transducer matrix.

\subsection{Proposed Simulation Framework}

In this section, we propose a 3D ultrasound simulation framework, based on the extension of Meunier et al. approach [12] in 3D. This model assumes that the point spread function (PSF) $H(x, y, z)$ of the transducer is linear and positionindependent, leading to formation of the radio-frequency ( $\mathrm{RF})$ signal $R F(x, y, z)$ via convolution of the system PSF with the impulse response $T(x, y, z)$ of the tissue:

$$
R F(x, y, z)=H(x, y, z) * T(x, y, z)
$$




\subsection{Image Formation Model}

The far-field PSF was approximated by a cosine function combined with a 3-D Gaussian envelope:

$$
H(x, y, z)=\exp \left(-\frac{1}{2}\left(\frac{x^{2}}{s_{x}^{2}}+\frac{y^{2}}{s_{y}^{2}}+\frac{z^{2}}{s_{z}^{2}}\right)\right) \cos (2 \pi f z)
$$

where $\left(s_{x}^{2}, s_{y}^{2}, s_{z}^{2}\right)$ define the size of the PSF in lateral, elevation and axial directions, respectively, and $f$ defines the transducer spatial frequency. This model assumes that time gain compensation was applied to correct for depth-dependent attenuation of the echos. It is also important to note that this model neglects several points: multiple scattering, multiple simultaneous interrogating directions, dispersion and attenuation phenomena and velocity variations.

\subsection{Scattering Modeling of the Tissues}

The tissue function $T(x, y, z)$ represents the scattering structure of the medium and can be related to the continuum tissue structure via the Laplacian operator applied to the compressibility distribution $B(x, y, z): T(x, y, z)=\Delta(B(x, y, z))$. Bamber and Dickinson in [3] proposed the following function $T(x, y, z)$ describing tissue echogeneity of a weakly inhomogeneous continuum in the far-field of an ultrasound beam, formed by plane-waves propagating in the $z$-direction:

$$
T(x, y, z)=\frac{1}{4} \frac{\partial^{2}}{z^{2}}\left(\frac{\rho(x, y, z)}{\rho_{0}}-\frac{\beta(x, y, z)}{\beta_{0}}\right)
$$

where $\rho$ and $\beta$ are the density and compressibility functions, with reference values $\rho_{0}$ and $\beta_{0}$. Seggie suggested in [19] to modify this model and replace the compressibility distribution with an impedance distribution $Z_{3 D}(x, y, z)$, arguing that for weak scattering, backscattering are mainly due to fluctuation in impedance. Pursuing this approach, Meunier et al. [12 proposed to model the impedance function with a common kernel function $C(x, y, z)$ and a "echogeneity" parameter $a_{n}$, which is proportional to the number of scatterers in a pixel:

$$
\begin{array}{r}
T(x, y, z)=\frac{\partial^{2}}{z^{2}} Z_{3 D}(x, y, z), \quad Z_{3 D}(x, y, z)=\sum_{n} Z_{n}(x, y, z), \\
Z_{n}(x, y, z)=a_{n} C_{3 D}\left(x-x_{n}, y-y_{n}, z-z_{n}\right) \\
C_{3 D}(x, y, z)=\exp \left(\frac{-1}{2}\left(\frac{x^{2}}{\sigma_{x}^{2}}+\frac{y^{2}}{\sigma_{y}^{2}}+\frac{z^{2}}{\sigma_{z}^{2}}\right)\right)
\end{array}
$$

The parameters $\left(\sigma_{x}, \sigma_{y}, \sigma_{z}\right)$ model the tissue cell dimensions and control the modeling of tissue anisotropy while $a_{n}$ controls the modeling of scatterer density within scanned pixels, which depends on both the transducer and the tissue. Because of important undersampling in the receiving mode of RT3D ultrasound 
transducers, assumption of large scatterers density (above 5 per pixel) can certainly be made in our simulation framework, leading to typical Rayleigh distributed RF signals as discussed in [12].

\subsection{Model Simplification}

In a $2 \mathrm{D}$ simulation framework, assuming that the diffraction angle in the elevation direction $y$ (i.e. the slice thickness) is very small, Meunier et al. in [12] further separated the transducer PSF in separated components $H(x, y, z)=H(x, z) \times H(y)$. Combined with the approximation of the tissue function as the $2 \mathrm{D}$ derivative, in the axial direction, of a 3D tissue function, they obtained a $2 \mathrm{D}$ RF image formation model in the image plane $(x, z)$. In this work, we did not use typical $2 \mathrm{D}$ transducer parameters to set our acquisition parameters for a RT3D transducer, as done in previous studies. Instead, we used available parameters from the commercial Volumetrics machine from Duke University [26] and estimated unknown parameters from sample RT3D ultrasound data. To approximate the actual image resolution of commercial RT3D ultrasound transducers, we set the PSF parameters to $(1,2,0.5) \mathrm{mm}$ for Full Width Half Maximum (FWHM) dimensions in axial, lateral, and transverse directions. The corresponding PSF parameters $\left(s_{x}^{2}, s_{y}^{2}, s_{z}^{2}\right)$ can be derived using the relationship $F W H M=2.35 \times$ standard deviation as used in [12. The transducer frequency was set to $3 \mathrm{MHz}(2.5-3.5 \mathrm{MHz}$ can be used with the Volumetrics transducer). The velocity of the acoustic wave in the human body was set to the standard $1540 \mathrm{~m} / \mathrm{s}$ average value.

In medical ultrasound systems, $H(x, y, z)$ is a narrowband signal. In this context, the second order derivative operator in Eq 3 can be discarded since the second derivative of a narrow-band is almost identical to the shape to the signreversed signal itself. This assumption is also used to simplify the model in other studies, explicitly in [10] or implicitly in [1230.

\subsection{Myocardium and Blood Cell Kernels Parameters Setting}

We chose a 3D Gaussian to model the impedance inhomogeneities as in [12]. Thus, the general cell kernel function was expressed as a Gaussian function with $\left(\sigma_{x}, \sigma_{y}, \sigma_{z}\right)=(10,10,35) \mu m$ given the fact that myocardial cells are elongated with dimensions around $(20,20,70) \mu \mathrm{m}$. Since myocardial cells are tightly connected and the myocardial fibers are parallel to each other at a scale of 100 or $1000 \mu m$, a (1:1:3.5) ratio can be observed at the image pixel scale. Recent studies on $2 \mathrm{D}$ or $3 \mathrm{D}$ ultrasound simulations only included modeling of the myocardium tissue. Since in actual clinical RT3D echocardiography, both myocardium and blood are imaged, we also built a tissue model for the blood. The average size of the blood cells is about $(10,10,2) \mu \mathrm{m}$. However, since blood cells are not tightly connected to each others and pixel volumes are much larger than individual blood cell volumes, the echogeneity within each blood pixel is mainly determined by the number of cells within each pixel, a random variable with Poisson distribution. We used $a_{n}=10$ or 30 to generate two volumes with different contrast between blood and myocardium, as illustrated in Fig. 3. The simulation with the highest density gave more satisfactory visual ultrasound data appearance. 

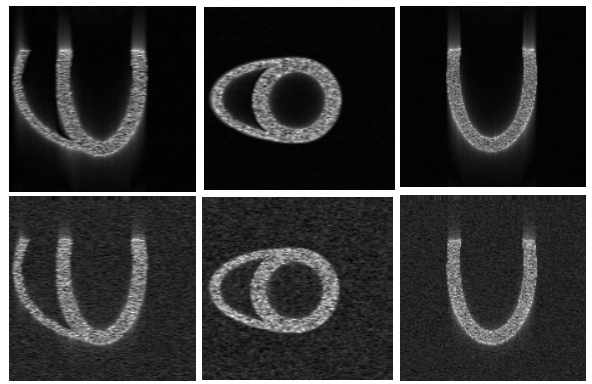

Fig. 3. Orthogonal views of a $3 \mathrm{D}$ ultrasound simulated volume of data: (top) low density of scatterers in blood pool, (bottom) high density

\section{Results and Discussion}

We generated two sets of simulations for the normal and pathological (LBBB) cases, varying the echogeneity parameters of the blood. These simulations provided very realistic volumes of images as illustrated in Fig. 3. We ran our OF tracking algorithm on the simulated dataset with the highest blood density, to compute the displacement vector field $\boldsymbol{u}$ on the endocardial and epicardial surfaces, initialized by the geometry of the first frame of the cardiac model. For computational efficiency, challenged by the very high spatial resolution of the simulated ultrasound data, we downsampled the point density on the myocardial surfaces by $50^{(1 / 3)}$ in each dimension, tracking about 500 points. Maximum displacement values, from the model, between two consecutive frames was $13 \mathrm{~mm}$ and rms angular displacements between consecutive frames between 10 and 75 deg. Accuracy of the motion field on the endocardial surface was computed via magnitude and angular errors over the whole cardiac cycle, as illustrated in Fig. 4. Epicardial tracking errors were very similar.

We also computed displacement maps from the true and estimated motion fields between the first frame (initiation of the depolarization) and end-systole (ES), after projection of the displacement vector field $\boldsymbol{u}$ in a cylindrical coordinate system $(r, \theta, z)$, with the $z$ axis oriented along the long axis of the ventricle. In this coordinate system, and using the AHA 16-sectors partition of the LV, we illustrate in Fig. 4 the circumferential component $u_{\theta}$, the radial component $u_{r}$ and the twisting $\partial u_{\theta} / \partial z$ of the displacement vector field $\boldsymbol{u}$ of the myocardium. Computation of these maps required tracking of the endocardial and epicardial surfaces, spatial interpolation of the motion field with radial-basis functions as described in [6] and correction for global translation of the myocardium between the first frame and ES. Note that the twist values, being computed as derivatives, need to be rescaled with respect to the sectorial height for physical values, which averages $20 \mathrm{~mm}$. End-diastole corresponded to frame 4 or 5 . We observed that maximum error measurements occurred at end-systole (i.e. frame 10). We also observed that specific displacement maps were very similar between the model and the OF estimations on our simulated data. Motion patterns between the 

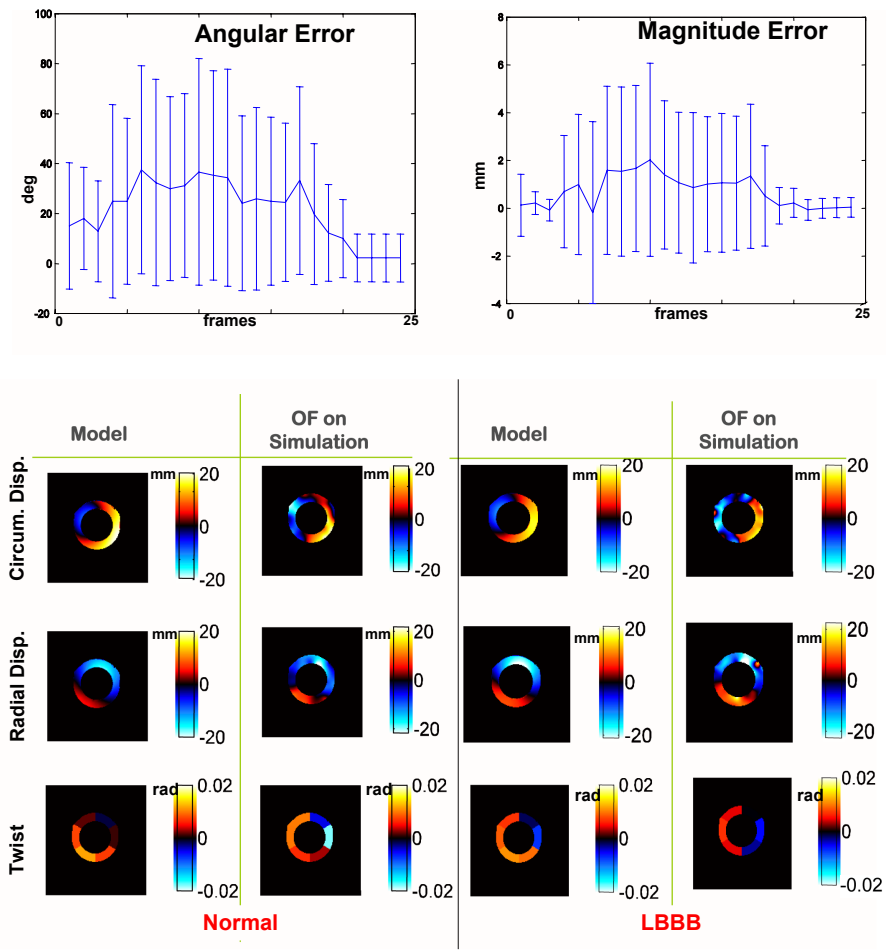

Fig. 4. Evaluation of displacements computed from optical flow (OF) on simulated RT3D ultrasound data: (top) Angular and magnitude error measurements on the endocardial surface. (bottom) Dense myocardial displacement fields from OF tracking of the myocardial surfaces.

normal and disease case were quite similar, as expected, since LBBB only incurs delayed contractions of the LV.

Regarding motion estimation errors, in a recent study, 24, the authors evaluated their OF method on simulated 2D ultrasound B-mode images, with Rayleigh distributions, computed as the modulus of complex RF signals. Motion was simulated on a short-axis like slices, with area-preserving radial displacements and a global translation. The overall velocity was set to a maximum of 2 pixels (versus 13 pixels for us). Results showed accuracy of angular estimates between $2.5^{\circ}$ and $5^{\circ}$, depending on the image SNR, and magnitude accuracy between $7 \%$ and $10 \%$. We are below such accuracy but we remain within an average error of $2 \pm 4$ pixels, despite very large displacements between consecutive frames.

\section{Conclusions}

Additional experiments will be performed to evaluate the accuracy of OF on simulated data and evaluate the precision of $\mathrm{OF}$ displacement estimation in additional disease cases, such as LAD, incurring local abnormal contractions. 
Regarding data assimilation perspectives, we emphasize that, once the procedure has been validated using synthetic measurements such as described in this paper the remaining difficulties in processing real data will focus on modeling aspects not parameter estimation nor image processing issues.

Acknowledgments. The authors would like to thank M. Alba (INRIA) for his contribution in generating the model simulation outputs.

\section{References}

1. Angelini, E., Homma, S., Pearson, G., Holmes, J., Laine, A.: Segmentation of realtime three-dimensional ultrasound for quantification of ventricular function: a clinical study on right and left ventricles. Ultrasound in Medicine and Biology 31(9), 1143-1158 (2005)

2. Angelini, E., Laine, A., Takuma, S., Holmes, J., Homma, S.: LV volume quantification via spatio-temporal analysis of real-time 3D echocardiography. IEEE Transactions on Medical Imaging 20(6), 457-469 (2001)

3. Bamber, J.C., Dickinson, R.J.: Ultrasonic b-scanning: a computer simulation. Physics in Medicne and Biology 25, 463-479 (1980)

4. Bresenham, V.: Algorithm for computer control of a digital plotter. IBM Systems Journal 4(1), 25-30 (1965)

5. Clifford, L., Fitzgerald, P., James, D.: Non-rayleigh first-order statistics of ultrasonic backscatter from normal myocardium. Ultrasound in Medicine and Biology 19(6), 487-495 (1993)

6. Duan, Q., Angelini, E., Gerard, O., Homma, S., Laine, A.: Comparing optical-flow based methods for quantification of myocardial deformations on rt3d ultrasound. In: IEEE International Symposium on Biomedical Imaging, Arlington, VA, USA, pp. 173-176 (2006)

7. Duan, Q., Angelini, E.D., Herz, S.L., Ingrassia, C.M., Gerard, O., Costa, K.D., Holmes, J.W., Laine, A.F.: Dynamic cardiac information from optical flow using four dimensional ultrasound. In: International Conference of the IEEE Engineering in Medicine and Biology Society (EMBS), pp. 4465-4468 (2005)

8. Duan, Q., Angelini, E.D., Herz, S.L., Ingrassia, C.M., Gerard, O., Costa, K.D., Holmes, J.W., Laine, A.F.: Evaluation of optical flow algorithms for tracking endocardial surfaces on three-dimensional ultrasound data. In: SPIE International Symposium, Medical Imaging San Diego, CA, USA (2005)

9. Jensen, J.A.: Field: A program for simulating ultrasound systems. Medical \& Biological Engineering \& Computing 34(1.1), 351-353 (1996)

10. Kallel, F., Bertrand, M., Meunier, J.: Speckle motion artifact under tissue rotation. IEEE Transactions on Ultrasonics, Ferroelectrics and Frequency Controls 41, 105-122 (1994)

11. Meunier, J.: Tissue motion assessment from 3d echographic speckle tracking. Physics in Medicine and Biology 43, 1241-1254 (1998)

12. Meunier, J., Bertrand, M.: Echographic image mean gray level changes with tissue dynamics: A system-based model study. IEEE Transactions on Biomedical Engineering 42(4), 403-410 (1995)

13. Meunier, J., Bertrand, M.: Ultrasonic texture motion analysis: theory and simulation. IEEE Transactions on Medical Imaging 14(2), 293-300 (1995) 
14. Narayanan, V.M., Shankar, P.M., Reid, J.M.: Non-rayleigh statistics of ultrasonic backscattered signals. IEEE Transactions on Ultrasonics, Ferroelectrics and Frequency Control 41(6), 845-852 (1994)

15. Neu, J.C., Krassowska, W.: Homogenization of syncytial tissues. Crit. Rev. Biomed. Eng. 21, 137-199 (1993)

16. Papademetris, X., Sinusas, A.J., Dione, D.P., Duncan, J.S.: Estimation of 3D left ventricular deformation from echocardiography. Medical Image Analysis 8, 285-294 (2004)

17. Sainte-Marie, J., Chapelle, D., Cimrman, R., Sorine, M.: Modeling and estimation of the cardiac electromechanical activity. Computers and Structures 84, 1743-1759 (2006)

18. Sarti, A., Bassi, P., Lamberti, C.: 3D modeling of phased array generated ultrasounds in lossy media. Computerized Medical Imaging and Graphics 17(4-5), 339-343 (1994)

19. Seggie, D.A., Leeman, S., Burge, R.E.: Realistic simulation of b-scan images. In: IEEE Ultrasonics Symposium, pp. 714-717 (1983)

20. Sermesant, M., Forest, C., Pennec, X., Delingette, H., Ayache, N.: Deformable biomechanical models: Application to $4 \mathrm{~d}$ cardiac image analysis. Medical Image Analysis 7(4), 475-488 (2003)

21. Sermesant, M., Moireau, P., Camara, O., Sainte-Marie, J., Andriantsimiavona, R., Cimrman, R., Hill, D.L., Chapelle, D., Razavi, R.: Cardiac function estimation from mri using a heart model and data assimilation: Advances and difficulties. Medical Image Analysis 10(4), 642-656 (2006)

22. Smith, S.W., Pavy, H.G., Von Ramm, O.T.: High speed ultrasound volumetric imaging system-part I: Transducer design and beam steering. IEEE Transactions on Ultrasonics, Ferroelectrics and Frequency Control 38(2), 100-108 (1991)

23. Smith, S.W., Ramm, O.T.V.: Real time volumetric ultrasound imaging system. Journal of Digital Imaging 3, 261-266 (1990)

24. Suhling, M., Arigovindan, M., Jansen, C., Hunziker, P., Unser, M.: Myocardial motion analysis from b-mode echocardiograms. IEEE Transactions on Image Processing 14, 525-553 (2005)

25. Tuthill, T.A., Sperry, R.H., Parker, K.J.: Deviations from rayleigh statistics in ultrasonic speckle. Ultrasonic Imaging 10(2), 81-89 (1988)

26. Von Ramm, O., Smith, S., Pavy, H.G.: High-speed ultrasound volumetric imaging system part II: parallel processing and image display. IEEE Transactions on Ultrasonics, Ferroelectrics and Frequency Control 38(2), 109-115 (1991)

27. Wagner, R.F., Insana, M.F., Brown, D.G.: Statistical properties of radiofrequency and envelope-detected signals with applications to medical ultrasound. Journal of the Optical Society of America 4, 910-922 (1987)

28. Yen, J.T., Smith, S.W.: Real-time rectilinear volumetric imaging. IEEE Transactions on Ultrasonics, Ferroelectrics and Frequency Control 49(1), 114-124 (2006)

29. Yen, J.T., Steinberg, J.P., Smith, S.W.: Sparse 2D array design for real-time rectilinear volumetric imaging. IEEE Transactions on Ultrasonics, Ferroelectrics and Frequency Control 47(1), 93-110 (2000)

30. Yu, W., Yan, P., Sinusas, A.J., Thiele, K., Duncan, J.S.: Towards pointwise motion tracking in echocardiographic image sequences: Comparing the reliability of different features for speckle tracking. Medical Image Analysis 10, 495-508 (2006) 